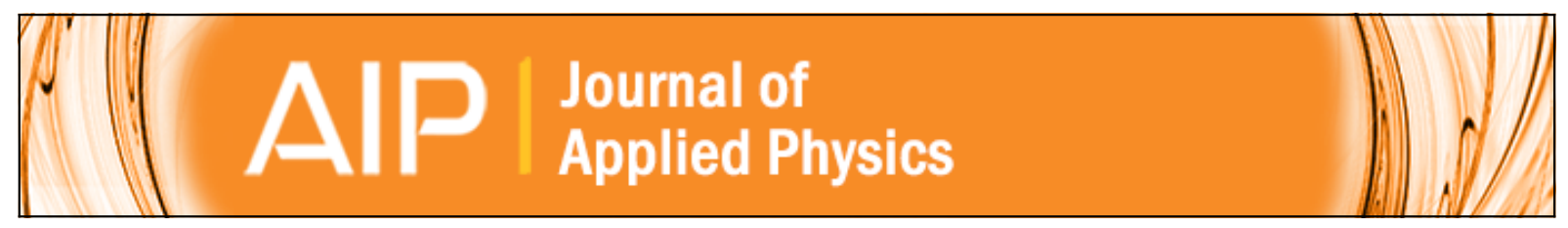

Comment on "Thickness dependence of exchange bias and coercivity in a ferromagnetic layer coupled with an antiferromagnetic layer" [J. Appl. Phys.94, 2529 (2003)]

J. Geshev, L. G. Pereira, and J. E. Schmidt

Citation: Journal of Applied Physics 96, 1763 (2004); doi: 10.1063/1.1767963

View online: http://dx.doi.org/10.1063/1.1767963

View Table of Contents: http://scitation.aip.org/content/aip/journal/jap/96/3?ver=pdfcov

Published by the AIP Publishing 


\title{
Comment on "Thickness dependence of exchange bias and coercivity in a ferromagnetic layer coupled with an antiferromagnetic layer" [J. Appl. Phys. 94, 2529 (2003)]
}

\author{
J. Geshev, L. G. Pereira, and J. E. Schmidt \\ Instituto de Física - UFRGS, 91501-970 Porto Alegre, RS, Brazil
}

(Received 20 October 2003; accepted 10 May 2004)

\begin{abstract}
$\mathrm{Hu}$, Jin, and Ma have proposed a theoretical investigation on the influence of the antiferromagnetic layer thickness on the magnetic properties of ferromagnetic/antiferromagnetic bilayers [J. Appl. Phys. 94, 2529 (2003)], considering both the bilinear and biquadratic exchange couplings, and have claimed that from their formulas for the hysteresis loop displacement and coercivity many interesting conclusions can be extracted. Unfortunately, the mathematical procedure used to find the equilibrium of the system is based on inadequate stability conditions and has led to nonphysical results. More importantly, the simple phenomenological model, employed by the authors, is intrinsically not capable to give the antiferromagnetic layer thickness dependence of the magnetic properties of such exchange-coupled bilayers. (c) 2004 American Institute of Physics.
\end{abstract}

[DOI: $10.1063 / 1.1767963$ ]

Recently Hu, Jin, and Ma have proposed [J. Appl. Phys. 94, 2529 (2003)] model calculations on the antiferromagnetic layer thickness dependence of exchange bias and coercivity in ferromagnetic (FM)/antiferromagnetic (AFM) bilayers. They used a generalized Meiklejohn-Bean ${ }^{1,2}$ (MB) model which assumes that the exchange-bias effect arises from the exchange coupling at an ideal uncompensated FM/AFM interface, considering the following total energy per unit interface area:

$$
\begin{aligned}
E= & K_{\mathrm{FM}} t_{\mathrm{FM}} \sin ^{2} \theta_{\mathrm{FM}}+K_{\mathrm{AFM}} t_{\mathrm{AFM}} \sin ^{2} \theta_{\mathrm{AFM}} \\
& -J_{E 1} \cos \left(\theta_{\mathrm{FM}}-\theta_{\mathrm{AFM}}\right)+J_{E 2} \cos ^{2}\left(\theta_{\mathrm{FM}}-\theta_{\mathrm{AFM}}\right) \\
& -H M_{\mathrm{FM}} t_{\mathrm{FM}} \cos \left(\theta_{\mathrm{H}}-\theta_{\mathrm{FM}}\right) .
\end{aligned}
$$

In the $\mathrm{Hu}$, Jin, and Ma notations, $K_{\mathrm{FM}}\left(K_{\mathrm{AFM}}\right)$ is the FM (AFM) uniaxial anisotropy constant, $t_{\mathrm{FM}}\left(t_{\mathrm{AFM}}\right)$ is the $\mathrm{FM}(\mathrm{AFM})$ layer thickness, $\theta_{\mathrm{FM}}\left(\theta_{\mathrm{AFM}}\right)$ is the angle formed by the FM (AFM) magnetic moment $\mathbf{M}_{\mathbf{F M}}\left(\mathbf{M}_{\mathbf{A F M}}\right)$ and the anisotropy axis (let us consider here that the FM and AFM easy axes coincide), $J_{E 1}$ and $J_{E 2}$ are the bilinear and biquadratic interfacial exchange-coupling terms, respectively, and $\mathbf{H}$ is the external magnetic field, applied at an angle $\theta_{\mathrm{H}}$ with respect to the easy axis.

Unfortunately, there are serious problems with the analytical expressions derived for the hysteresis loop displacement $H_{\mathrm{EB}}$ and the coercivity $H_{C}$ and, as a consequence, with the conclusions made.

To begin with, Hu, Jin, and Ma used an incorrect procedure to find the equilibrium magnetization state, which should be determined by equating the first partial derivatives of $E$ with respect to $\theta_{\mathrm{FM}}$ and $\theta_{\mathrm{AFM}}$ to zero,

$$
\frac{\partial E}{\partial \theta_{\mathrm{AFM}}}=\frac{\partial E}{\partial \theta_{\mathrm{FM}}}=0
$$

and the stability must be then examined with the aid of the conditions (see, for example, Refs. 3 and 4)

$$
\frac{\partial^{2} E}{\partial \theta_{\mathrm{FM}}^{2}} \frac{\partial^{2} E}{\partial \theta_{\mathrm{AFM}}^{2}}-\left(\frac{\partial^{2} E}{\partial \theta_{\mathrm{FM}} \partial \theta_{\mathrm{AFM}}}\right)^{2}>0
$$

and

$$
\frac{\partial^{2} E}{\partial \theta_{\mathrm{FM}}^{2}}>0 \text { or } \frac{\partial^{2} E}{\partial \theta_{\mathrm{AFM}}^{2}}>0 .
$$

Instead of using the above procedure, $\mathrm{Hu}$, Jin, and Ma, following Morrish ${ }^{5}$ (Ref. 20 in their paper), erroneously examined the conditions at which the second partial derivatives $\partial^{2} E / \partial \theta_{\mathrm{FM}}^{2}$ and $\partial^{2} E / \partial \theta_{\mathrm{AFM}}^{2}$ vanish. Such a treatment, however, is not appropriate for the case of two variables and consequently the expressions, obtained for $H_{\mathrm{EB}}$ and $H_{C}$, are not valid.

Moreover, the authors found two rather complex expressions of $H_{\mathrm{EB}}$ and $H_{C}$, both containing $J_{E 1}$ and $J_{E 2}$. It is very easy to see, however, that these formulas must not depend on $J_{E 1}$ and $J_{E 2}$ (the two-equation system formed by the sum of the first derivatives [Eqs. (2) and (3) in their paper] and the difference between the second ones [their Eqs. (4) and (5)] does not contain any exchange-coupling constant). Thus, the critical fields, and consequently the expressions for $H_{\mathrm{EB}}$ and $H_{C}$, should not depend on the coupling in their treatment.

Using the correct equilibrium conditions [Eqs. (2)-(4) in the present Comment] one can derive the corresponding expressions in the framework of this generalized MB model, as it has been done for the AFM domain-wall formation case. ${ }^{4}$ Although the detailed deduction of these formulas [which will give more than one $\left(H_{C}, H_{\mathrm{EB}}\right)$ pair, depending on the ratio between the AFM anisotropy and the coupling constant strengths, ${ }^{4}$ i.e., strong, intermediate, and weak coupling] is not done here since it is not the aim of the present Comment, an example can be shown. With the use of the normalized parameters $K_{\mathrm{AFM}}^{\prime}=K_{\mathrm{AFM}} t_{\mathrm{AFM}} / t_{\mathrm{FM}}, \quad J_{E 1}^{\prime}=J_{E 1} / t_{\mathrm{FM}}, \quad$ and $J_{E 2}^{\prime}$ $=J_{E 2} / t_{\mathrm{FM}}$, one obtains 


$$
H_{C}=\frac{2 K_{\mathrm{FM}}}{M_{\mathrm{FM}}}-\frac{2 K_{\mathrm{AFM}}^{\prime}\left[J_{E 2}^{\prime}\left(K_{\mathrm{AFM}}^{\prime}-J_{E 2}^{\prime}\right)+J_{E 1}^{\prime 2} / 4\right]}{M_{\mathrm{FM}}\left[\left(K_{\mathrm{AFM}}^{\prime}-J_{E 2}^{\prime}\right)^{2}-J_{E 1}^{\prime 2} / 4\right]}
$$

and

$$
H_{\mathrm{EB}}=\frac{J_{E 1}^{\prime}}{M_{\mathrm{FM}}\left[\left(1-J_{E 2}^{\prime} / K_{\mathrm{AFM}}^{\prime}\right)^{2}-J_{E 1}^{\prime 2} /\left(2 K_{\mathrm{AFM}}^{\prime}\right)^{2}\right]}
$$

for weak exchange coupling $\left(K_{\mathrm{AFM}}^{\prime}\right.$ high as compared to $J_{E 1}^{\prime}$ and/or $J_{E 2}^{\prime}$ ). One can see that, contrary to the plots in Fig. 2 in the $\mathrm{Hu}$, Jin, and Ma paper, $H_{C}$ rises towards the bulk FM layer coercivity $\left(=2 K_{\mathrm{FM}} / M_{\mathrm{FM}}\right)$ and $H_{\mathrm{EB}}$ decreases asymptotically to $J_{E 1} /\left(M_{\mathrm{FM}} t_{\mathrm{FM}}\right)$ with the $K_{\mathrm{AFM}}^{\prime}$ increase, as expected. $^{1,2}$

We also checked the possibility of the mixed derivative in Eq. (3) to be zero, in which (very particular) case the $\mathrm{Hu}$, Jin, and Ma treatment could, hypothetically, give some meaningful result. Unfortunately, even though there exists a mathematical solution, it has no physical meaning.

More importantly, it must be emphasized that the simple model employed by the authors in the paper under discussion, as well as in at least one more paper of theirs [J. Appl. Phys. 92, 1009 (2002)], is intrinsically not capable to give the antiferromagnetic layer thickness dependence of the magnetic properties of exchange-coupled systems. Phenomenologically, the MB energy per unit volume of the FM, $E^{\prime}=E / t_{\mathrm{FM}}$,

$$
\begin{aligned}
E_{\mathrm{MB}}^{\prime}= & K_{\mathrm{FM}} \sin ^{2} \theta_{\mathrm{FM}}+K_{\mathrm{AFM}}^{\prime} \sin ^{2} \theta_{\mathrm{AFM}} \\
& -J_{E 1}^{\prime} \cos \left(\theta_{\mathrm{FM}}-\theta_{\mathrm{AFM}}\right)+J_{E 2}^{\prime} \cos ^{2}\left(\theta_{\mathrm{FM}}-\theta_{\mathrm{AFM}}\right) \\
& -H M_{\mathrm{FM}} \cos \left(\theta_{\mathrm{H}}-\theta_{\mathrm{FM}}\right)
\end{aligned}
$$

represents the energy of two exchange-coupled magnetic moments, $\mathbf{M}_{\mathbf{F M}}$ and $\mathbf{M}_{\mathbf{A F M}}$, characterized by uniaxial anisotropies with constants $K_{\mathrm{FM}}$ and $K_{\mathrm{AFM}}^{\prime}$, respectively, being only $\mathbf{M}_{\mathbf{F M}}$ subjected to a field $\mathbf{H}$.

It is worth to recall that the $\mathrm{MB}$ model assumes unequal numbers of antiparallel AFM moments at the interface or, in other words, that the AFM plane adjacent to the interface is a plane of parallel moments, with total magnetic moment $\mathbf{M}_{\mathbf{A F M}}$, providing the torque exerted on $\mathbf{M}_{\mathbf{F M}}$, which is essential to the exchange-bias effect. Once formed this interface AFM plane, its thickness, in practice, cannot be changed. Thus, the variation of the parameter $t_{\mathrm{AFM}}$, investigated by $\mathrm{Hu}$, Jin, and $\mathrm{Ma}$, will actually vary the value of $K_{\mathrm{AFM}}^{\prime}\left(=K_{\mathrm{AFM}} t_{\mathrm{AFM}} / t_{\mathrm{FM}}\right)$, i.e., any increase of $K_{\mathrm{AFM}}^{\prime}$ could be attributed to an increase of the AFM anisotropy constant or to a decrease of $t_{\mathrm{FM}}$, but not to a raise of the thickness of the AFM interface layer.

Doubtlessly, the total thickness of the antiferromagnet and the crystalline quality of the latter influence its properties, determining its magnetic state (e.g., antiferromagnetic, paramagnetic or superparamagnetic one, especially for low thicknesses, when finite-size effects can take place), its blocking temperature, training effects, the strength and/or the type of the AFM anisotropy, etc. A number of studies can be found in the literature on this subject. It is impossible, however, to account for this influence employing the MB model in the manner used in the paper under discussion.

In summary, we showed that the analytical expressions for the hysteresis loop shift and coercivity derived by $\mathrm{Hu}$, Jin, and Ma are untrustworthy. It is particularly important to emphasize that the model used by the authors is not capable to give the dependence of the magnetic properties of such exchange-coupled bilayers on the total antiferromagnetic layer thickness.

This work was supported by CNPq, Brazil.

${ }^{1}$ W. H. Meiklejohn and C. P. Bean, Phys. Rev. 105, 904 (1957).

${ }^{2}$ W. H. Meiklejohn, J. Appl. Phys. 33, 1328 (1962).

${ }^{3}$ M. R. Spiegel, Theory and Problems of Advanced Calculus (McGraw-Hill, New York, 1965), Chap. 8, p. 164.

${ }^{4}$ J. Geshev, Phys. Rev. B 62, 5627 (2000).

${ }^{5}$ A. H. Morrish, The Physical Principles of Magnetism (Wiley, New York, 1965), Chap. 7 p. 344. 